First publ. in: Physical Review B, Vol. 72 (2005), Article 024501

\title{
Shot-noise and conductance measurements of transparent superconductor/two-dimensional electron gas junctions
}

\author{
B.-R. Choi, A. E. Hansen, T. Kontos, C. Hoffmann, S. Oberholzer, W. Belzig, and C. Schönenberger* \\ Institute of Physics, University of Basel, Klingelbergstr. 82, CH-4056 Basel, Switzerland \\ T. Akazaki and H. Takayanagi \\ NTT Basic Research Laboratories, 3-1 Morinosato-Wakamiya, Atsugi-shi, Kanagawa 243-0198, Japan \\ (Received 30 September 2004; revised manuscript received 10 January 2005; published 1 July 2005)
}

\begin{abstract}
We have measured the conductance and shot noise of superconductor-normal metal (S-N) junctions between a niobium $(\mathrm{Nb})$ film and a two-dimensional electron gas (2DEG), formed in an InAs-based semiconductor heterostructure. Adjacent to the junction, the 2DEG is shaped into a sub-micrometer beam splitter. The current shot noise measured through one arm of the beam splitter is found to be enhanced due to Andreev reflection. Both noise and conductance measurements indicate that the Nb-2DEG interface is of high quality with a transparency approaching $\approx 60-70 \%$. The present device can be seen as a quasi-ballistic S-N beam-splitter junction.
\end{abstract}

PACS number(s): 74.78.Na, 73.23. $-\mathrm{b}, 74.45 .+\mathrm{c}, 72.70 .+\mathrm{m}$

\section{INTRODUCTION}

Shot-noise measurements provide a powerful tool to study charge transport in mesoscopic systems. ${ }^{1}$ Whereas resistance measurements yield information on the average probability for the transmission of electrons from source to drain, shot noise provides additional information on the electron transfer process, which cannot be obtained from resistance measurements. For instance, the charge of quasiparticles can be extracted from shot-noise measurements, an experiment that was applied to the fractional quantum Hall regime. ${ }^{2-4}$ Shot noise also provides information on the statistics of the electron transfer. In general, the fermionic nature of the particles lead to a suppression of the shot noise from its classical value $S_{I}=2 e|I|$, corresponding to Poissonian statistics $\left(S_{I}\right.$ is the power-spectral density of current fluctuations in units of $\left.\mathrm{A}^{2} \mathrm{~s}\right)$. Suppression can also be induced by Coulomb interaction, which was observed in the single-electron tunneling regime. ${ }^{5}$ That shot noise can be fully suppressed in an open channel was confirmed in quantum-point contacts. ${ }^{6,7}$ In a general conductor, the suppression is not full, but depends on the actual distribution of transmission eigenvalues. ${ }^{8-10}$ For example, shot noise is suppressed to $1 / 3$ in a disorder wire ${ }^{11-16}$ and to $1 / 4$ in an open cavity. ${ }^{17-20}$ For a recent review, see Ref. 21.

Different to mesoscopic devices with normal electron reservoirs, shot noise can be enhanced in devices with superconducting leads by virtue of the Andreev reflection process taking place at the interface between a normal metal and a superconductor. ${ }^{22-29}$ In some limiting cases, e.g., in the tunneling and disordered limit, the shot noise can be doubled with respect to its normal state value. ${ }^{8,30-34}$

In addition to measure shot noise in a two-terminal geometry, multiterminal fluctuation measurements have been proposed. ${ }^{35}$ Whereas shot noise corresponds to the autocorrelation of fluctuations, cross-correlation measurements of fluctuations between different leads provide a wealth of new experiments. As pointed out by Büttiker, exchange correla- tions can, for example, be measured directly. ${ }^{35} \mathrm{In}$ an attempt to go beyond conventional shot-noise measurements, correlation measurements ${ }^{36,37}$ on electron beam splitters ${ }^{38}$ were studied. The partitioning of a "stream" of ferminons in a beam splitter results in negative correlations between the fluctuations measured on the two output ports (antibunching). In contrast, bunching-like behavior (positive correlation) has theoretically been predicted in multiterminal devices in which at least one electrode is a superconductor. ${ }^{39-42}$ In the subgap region, charge is injected from the superconducting lead into the device in correlated pairs of electrons, which - in the simplest picture-may separate in the normal scattering region and exit at two different leads. As a consequence, the current in the exit leads fluctuate in parallel. However, it has been pointed out that this picture is misleading, in particular in the regime where the superconductor is strongly coupled to the normal region. In this case, the normal region should rather be viewed as a proximity-induced superconductor. ${ }^{43}$ Positive correlations have not been observed in mesoscopic devices until today.

Finally, we mention that the experimental quest for positive correlations is also important for the new field of quantum computation and communication in the solid state, ${ }^{44,45}$ in which entangled electrons play a crucial role. A natural source of entanglement is found in superconductors in which electrons are paired in a spin-singlet state. A source of entangled electrons may therefore be based on a superconducting injector. ${ }^{42,46-54}$ Even more so, an electronic beam splitter is capable of distinguishing entangled electrons from single electrons. ${ }^{55,56}$

Motivated by all this theoretical work, we decided to fabricate superconductor-normal metal (S-N) devices composed of a superconducting injector $(\mathrm{Nb})$ into a high-mobility InAs-based two-dimensional electron gas (2DEG), ${ }^{57-62}$ in which beam splitters can be fabricated. In this paper, we focus on the fabrication of such devices in Sec. II and their electrical characterization in terms of linear and nonlinear resistance in Sec. III A, as well as shot noise in Sec. III B. 
(a)
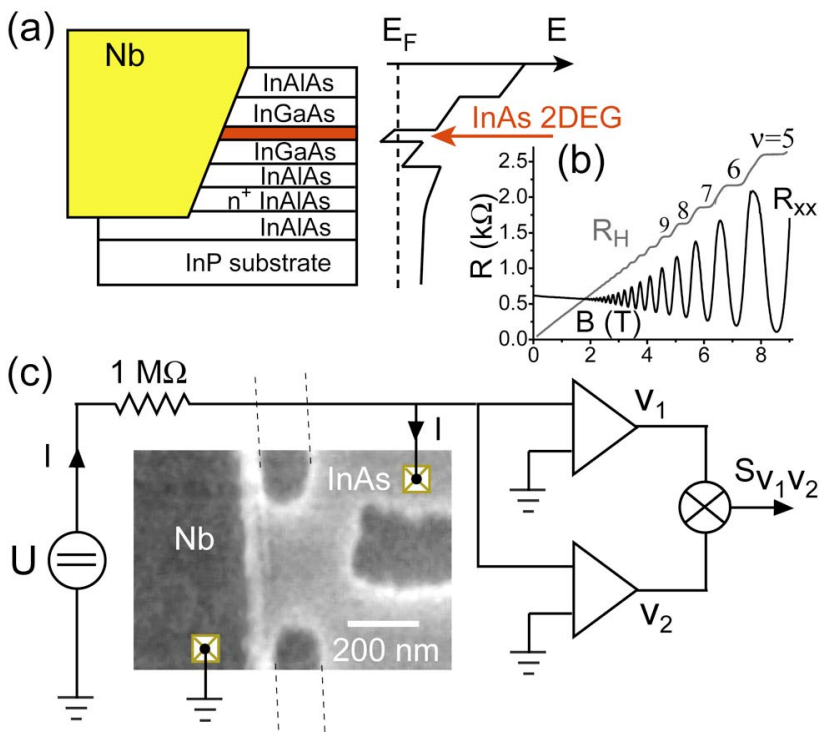

FIG. 1. (a) Schematic side view of the Nb contact to the InAs semiconductor heterostructure and the corresponding energy band diagram. (b) Longitudinal $\left(R_{x x}\right)$ and Hall $\left(R_{H}\right)$ resistance measured on this heterostructure. (c) SEM picture of a sample (top view) with a diagram of the measurement setup. The sample is current biased through a series resistor and the voltage fluctuations are measured with the aid of two sets of amplifiers whose outputs are cross correlated. $S$ denotes the power spectral density in units of $\mathrm{V}^{2} / \mathrm{Hz}$.

The shot noise of the current from the superconductor to one of the normal reservoirs is enhanced for bias currents corresponding to voltages below the superconducting gap of the $\mathrm{Nb}$ contact. This enhancement is due to Andreev reflections at the superconducting contact and disappears in a magnetic field higher than the critical field of $\mathrm{Nb}$. Using the coherent scattering theory, we extensively compare our measurements with different models in Sec. IV. Our devices can best be described as composed of a highly transparent S-N interface in series with a short scattering region, whose size $L$ is comparable to the elastic mean-free path $l_{e}$.

\section{EXPERIMENT}

The InAlAs/InGaAs heterostructure was grown by molecular beam epitaxy on a Fe-doped semi-insulating InP substrate. The $2 \mathrm{DEG}$ is confined in a $4 \mathrm{~nm}$ wide InAs quantum well $35 \mathrm{~nm}$ below the surface of the heterostructure, see Ref. 57 and Fig. 1(a) for details. The substrate is first structured into a $50 \mu \mathrm{m}$ wide Hall bar (MESA) by wet etching. Hall and Shubnikov-de Haas measurements [Fig. 1(b)] then yield an electron density of $n_{e}=2.1 \cdot 10^{16} \mathrm{~m}^{-2}$ and a mobility of $\mu=5.0 \mathrm{~m}^{2} / \mathrm{Vs}$ for the $2 \mathrm{DEG}$, corresponding to a Fermi wavelength of $\lambda_{F}=18 \mathrm{~nm}$ and an elastic mean-free path of $l_{e}=1.2 \mu \mathrm{m}$.

The $\mathrm{Nb}$ electrode is defined by electron beam lithography at one side of the MESA. First, the MESA is etched in the patterned electrode area to a depth of $\sim 50 \mathrm{~nm}$. Then, the sample is mounted in an evaporation chamber and rf-sputter cleaned. Without breaking the vacuum, an $80 \mathrm{~nm}$ thick $\mathrm{Nb}$ film is subsequently deposited at an angle of $30 \mathrm{deg}$ to the horizontal. After liftoff, a $50 \mu \mathrm{m}$ wide superconductor2DEG contact is obtained. A cross section through such a $\mathrm{Nb}$ contact is schematically shown in Fig. 1(a).

E-beam lithography is now used to reduce the macroscopic superconductor-2DEG contact to submicron dimensions, see Fig. 1(c). This is achieved by etching trenches into the heterostructure to a depth of $60 \mathrm{~nm}$ below the surface, removing the conducting InAs quantum well. Three trenches are etched, two vertical ones and one horizontal one, which start at the nanometer-sized contact in front of the superconductor and extend across the whole MESA. The vertical trenches have a width of about $\sim 100 \mathrm{~nm}$ and are placed parallel and as close as possible to the $\mathrm{Nb}$ interface at a distance of less than $50 \mathrm{~nm}$. The three-terminal junction consist thereafter of a $300 \times 350 \mathrm{~nm}$ square area in the $2 \mathrm{DEG}$ which is bound on one side ( $350 \mathrm{~nm}$ wide) by the edge of the superconductor and the other side by two constrictions leading to two macroscopic normal electron reservoirs. The constrictions have a nominal width $w$ of $170 \mathrm{~nm}$, corresponding to $N=2 w / \lambda_{F} \approx 19$ conducting channels. This part can be viewed as a beam splitter for charge carriers (Cooper pairs in the superconducting state), injected from the $\mathrm{Nb}$ contact.

Many samples were fabricated and electrically analyzed with qualitatively identical results. In the following, we present the data of one sample, which was measured in a ${ }^{3} \mathrm{He}$ cryostat with a base temperature of $270 \mathrm{mK}$. Though correlation measurements are the long term goal of this work, we decided to focus on two-terminal shot-noise measurements first as schematically shown in Fig. 1(c), because the measurement signal (for technical reasons) is much higher in this type of measurement as compared to correlation measurements. The sample is current biased through a $1 \mathrm{M} \Omega$ series resistor thermally anchored at the $1 \mathrm{~K}$ pot of the cryostat. The current is determined by the dc bias voltage $U$, on which a small ac voltage is superimposed in order to measure the differential resistance $d V / d I$. All measurement lines are filtered at low temperature by lossy microcoax cables and additional $\pi$ filters are used at room temperature. Two ultralow-noise amplifiers (LI-75, NF Corp., Yokohama, Japan) with a fixed gain of 100, followed by two low-noise amplifiers (Stanford SR560, operated at a nominal gain of 10 or 100), are used to measure the voltage fluctuations across the sample in parallel. All amplifiers are operated at room temperature and powered by independent sets of batteries to minimize cross talk. The voltage signals from the amplifiers are then cross correlated by a spectrum analyzer (HP 89410A). This cross-correlation technique ${ }^{63}$ can eliminate (or greatly reduce) the voltage noise contributions due to the two amplifiers, because they ought to fluctuate in an uncorrelated manner.

In order to measure shot noise, which is a frequencyindependent contribution, one has to ensure that $1 / f$ noise can be neglected at the highest bias currents. As a consequence, we have measured the noise at rather large frequencies $f$ around $50-200 \mathrm{kHz}$. In this window, $1 / f$ noise can be neglected up to the highest currents of $\approx 2 \mu \mathrm{A}$. Due to capacitances in the whole circuit including the measurement lines, the signal is damped. The overall gain, including the frequency-dependent attenuation, has to be carefully calibrated for each device separately. This is done by measuring 
(a)

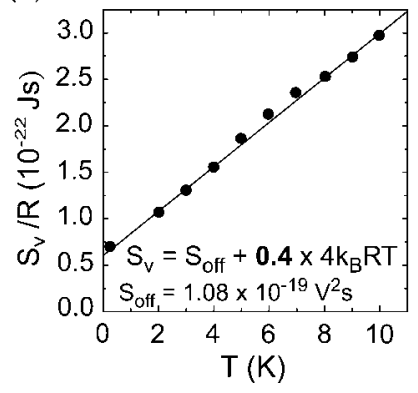

(b)

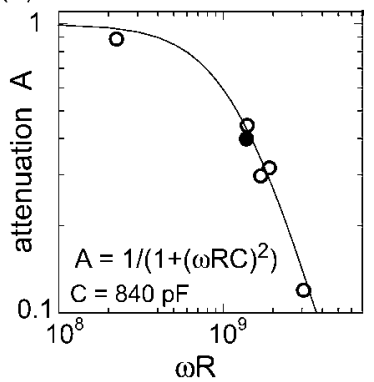

FIG. 2. (a) Example of a measurement of the equilibrium (thermal) voltage noise (spectral density $S_{V}$ ) versus temperature $T$ used to deduce the calibration parameters. Here, the frequency and sample resistance were $f=110 \mathrm{kHz}$ and $R \simeq 2 \mathrm{k} \Omega$, respectively. The thermal noise is linearly dependent on $T$ and the slope (Ref. 64) yields the attenuation factor $A$ of the signal. (b) The attenuation $A$ as a function of $\omega R$ follows the dependence expected for a simple RC network, i.e., $A(\omega)=\left[1+(\omega R C)^{2}\right]^{-1}$.

the equilibrium voltage noise (i.e., the thermal noise), given by $S_{V}=4 k_{B} T R$, as a function of temperature $T$, as shown in Fig. 2(a). Here, $k_{B}$ is the Boltzmann constant and $R$ is the linear-response sample resistance (more precisely, the parallel connection of the sample resistance with the series biasing resistor). The measured voltage noise $S_{V_{1}, V_{2}}$, including the amplifier noise, can be written as [see Fig. 1(c)]

$$
S_{V_{1}, V_{2}}=A(\omega) R^{2}\left(S_{I}+S_{I, \text { off }}\right)+S_{V, \text { off }} .
$$

Here, $S_{I}$ is the current noise of the sample, which in the calibration procedure is of thermal origin only, i.e., $S_{I}$ $=4 k_{B} T / R$. $S_{I, \text { off }}$ denotes the current noise offset of the two LI-75 amplifiers. This contribution cannot be eliminated by the cross-correlation scheme. We also find a nonzero voltage noise offset $S_{V \text {,off }}$ accounting for residual cross talk between the amplifiers, possibly due to spurious ground currents. The nominal overall gain of either $10^{3}$ or $10^{4}$ of the amplifiers has been divided off in the above Eq. (1). Hence, all the quantities refer to "input" noise. Finally, $A(\omega=2 \pi f)$ denotes the frequency-dependent attenuation factor.

A typical calibration measurement at $f=110 \mathrm{kHz}$ is shown in Fig. 2(a). The attenuation $A$ at this frequency is obtained from the slope of $S_{V}(T)$ and the residual amplifier noise from the vertical offset of the fitted linear dependence extrapolated to $T=0 .{ }^{64}$ The attenuation was measured for different devices with varying resistances $R$, ranging between $\approx 1.5$ and $\approx 2.5 \mathrm{k} \Omega$ and frequencies in the range of 50 to $\approx 200 \mathrm{kHz} . A(\omega)$ for a set of devices is shown in Fig. 2(b) to follow the expected damping for a simple (RC) network, i.e., $A(\omega)=\left[1+(\omega R C)^{2}\right]^{-1}$. The extracted capacitance of $C$ $=840 \mathrm{pF}$ is mainly due to the filtering of the wires (microcoax filters) and the two input capacitances of the amplifiers.

The noise offset $S_{\text {off }}$, extracted from the calibration procedure, typically amounts to $1 \cdot 10^{-19} \mathrm{~V}^{2} \mathrm{~s}$. The current noise of a single LI-75 amplifier is specified to be $<2 \cdot 10^{-28} \mathrm{~A}^{2} \mathrm{~s}$ and independently measured to be $<8 \cdot 10^{-28} \mathrm{~A}^{2} \mathrm{~s}$, corresponding to a voltage noise of $<3.2 \cdot 10^{-21} \mathrm{~V}^{2} \mathrm{~s}$ for a single amplifier on a typical sample resistor of $R=2 \mathrm{k} \Omega$, or to

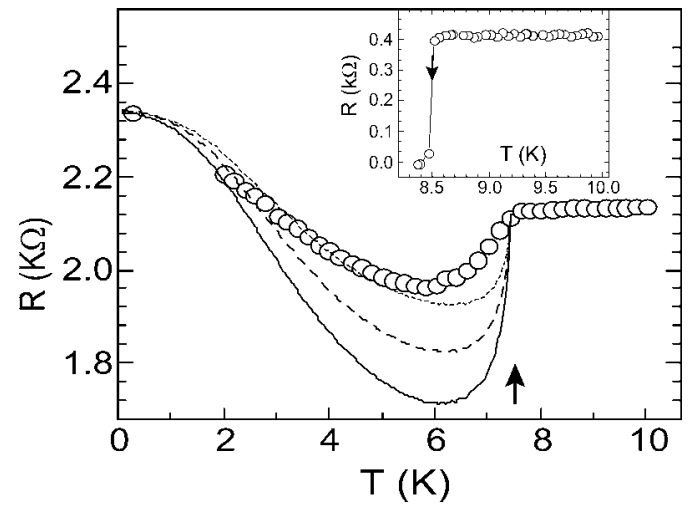

FIG. 3. Temperature dependent resistance $R(T)$. Circles correspond to the measurements, whereas the curves are calculated using the BTK model together with a classical series resistor $R_{S}=0$ (solid), $R_{S}=500$ (dashed), and $R_{S}=1000 \Omega$ (dotted). $\Delta$ was fixed to $1.14 \mathrm{meV}$ and the barrier transparency $\Gamma \approx 0.72$ and channel number $N \approx 9$ were deduced so that the theoretical $R(T)$ matches the experiment one in the normal state and the superconducting state at the lowest temperature. The inset shows the superconducting transition of the $\mathrm{Nb}$ film measured with two probes on a structured device. The transitions of the $\mathrm{Nb}$ film and the submicron $\mathrm{Nb}$ contact are marked by arrows.

$<6.4 \cdot 10^{-21} \mathrm{~V}^{2} \mathrm{~s}$ for two amplifiers in parallel. The offset current noise of the amplifiers is therefore at least an order of magnitude smaller than the measured offset and can therefore not account for it. Hence, the dominating part of the measured offset is caused by residual voltage fluctuations and we set the amplifier current offset to zero in the following. The voltage noise floor of a single LI-75 amplifier is specified to be $1.4 \cdot 10^{-18} \mathrm{~V}^{2} \mathrm{~s}$ and independently measured (short circuit input) to be $2.5 \cdot 10^{-18} \mathrm{~V}^{2} \mathrm{~s}$, a value which is substantially larger than the measured offset noise after the cross correlation. The cross correlation technique therefore reduces the voltage fluctuations of the amplifiers by as much as a factor of 25 .

The deduced calibration parameters are then used to extract the intrinsic current shot-noise $S_{I}$ generated in a superconducting-2DEG junction from the measured noise $S_{V_{1}, V_{2}}$ using Eq. (1). It is important to emphasize that $R$ in Eq. (1) has to be replaced by the differential resistance $d V / d I$ for the nonequilibrium measurement. This is crucial, because of the nonlinear current-voltage characteristic of these devices.

\section{RESULTS}

We measured the linear-response resistance $R$ as a function of temperature $T$, the differential resistance $d V / d I$, and the spectral density of the voltage fluctuations (the noise) as a function of bias current $I$, both at $T=270 \mathrm{mK}$. We focus first on the resistance and then on the noise measurements.

\section{A. Resistance measurements}

Figure 3 shows the temperature dependence of the linearresponse resistance $R$ measured from the superconductor to one of the normal reservoirs, as schematically shown in Fig. 
1(c). Above $T=7.5 \mathrm{~K}$, the resistance is constant, whereas it varies nonmonotonically below. $R$ first drops abruptly below $7.5 \mathrm{~K}$, has a minimum at $\approx 6 \mathrm{~K}$ and then starts to increase for lower temperatures. At the lowest temperature, $R$ is $\approx 8 \%$ higher than $R(T>7.5 \mathrm{~K})$. The drop at $7.5 \mathrm{~K}$ is identified with the superconducting transition temperature $T_{c}$ of the junction. The superconducting transition of the $\mathrm{Nb}$ film was also measured via two contacts bonded to the $\mathrm{Nb}$ electrode of the actual device. We found $T_{c}=8.5 \mathrm{~K}$ (inset of Fig. 3). A suppression of $T_{c}$ in a film from its bulk value of $9.5 \mathrm{~K}$ is commonly observed, as is a similar suppression of $T_{c}$ in microfabricated structures. The relative modest suppression of $\approx 1 \mathrm{~K}$ is in agreement with previous work, see for example Ref. 27.

The nonmonotonic temperature dependence, which we observe in Fig. 3, suggests that the superconductor-2DEG interface has an intermediate transparency. This is qualitatively deduced by referring to the Blonder-TinkhamKlapwijk (BTK) model of a superconductor-normal metal junction. ${ }^{65}$ In this model scattering is exclusively taking place at the junction interface described by a single parameter, which is the transmission probability (transparency) of the junction. This situation is referred to as the clean or ballistic junction limit (also the BTK limit), as opposed to the case in which additional scattering in the normal part of the junction is introduced. If the junction has a low transparency (tunnel junction), the resistance is expected to increase exponentially fast at low temperature. On the other hand, if the junction has a very high transparency, $R$ decreases monotonically to reach half of its normal state value at the lowest temperature. We neither see an exponential increase, nor a monotonic decrease of $R$, suggesting intermediate transparency.

In the following, if we refer to the normal state resistance $R_{N}$, we mean $R(\sim 8 \mathrm{~K})$, and if we refer to the resistance in the superconducting state $R_{S}$, we mean $R(270 \mathrm{mK})$.

The measured normal-state resistance $R_{N}$ of this device equals $2.13 \mathrm{k} \Omega$. It is straightforward to compare the corresponding normal-state conductance $G_{N}=R_{N}^{-1}$ with the Landauer formula, ${ }^{66}$ i.e., with $G_{N}=\left(2 e^{2} / h\right) N \Gamma$, where $N$ is the number of eigenchannels with nonzero transmission eigenvalues $\mathcal{T}_{n}$ and $\Gamma$ the mean value of $\mathcal{T}_{n}$. Taking $N$ to be 19 , as determined from the geometrical width of the constrictions, yields $\Gamma \approx 0.32$ as the average transmission coefficient of the entire device. The resistance can have contributions from both the superconductor-2DEG interface and the point contacts to the normal reservoirs. Therefore, $\Gamma \approx 0.32$ must be seen as a lower bound for the $\mathrm{S}-\mathrm{N}$ interface transparency. This will be studied in greater detail in Sec. IV.

In Fig. 3 are also shown calculated curves of $R(T)$ which were matched to agree simultaneously with $R_{N}$ and $R_{S}$. The solid curve corresponds to the BTK model for a junction transparency of $72 \%$. The minimum of $R(T)$ is much more pronounced in the calculated curve. In an attempt to account for additional scattering, for example, at the constrictions of the beam splitter, a classical series resistor was added (dashed and dotted curves). This clearly improves the overall matching, but strong deviations remain close to $T_{c}$. We mention that similar resistance values and temperature dependen-

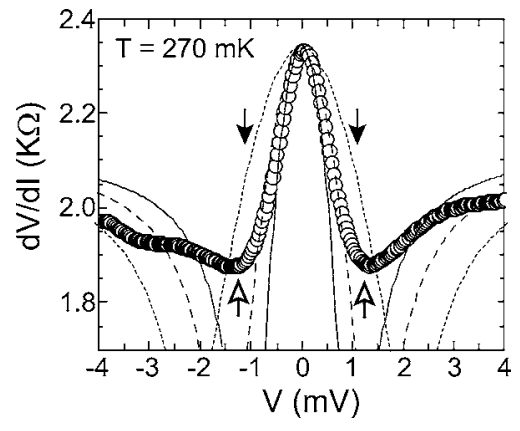

FIG. 4. Voltage dependent differential resistance $d V / d I(V)$ measured at $T=270 \mathrm{mK}$. Circles correspond to the measurements, whereas the curves are calculated using the BTK model together with a classical series resistor $R_{S}=0$ (solid), $R_{S}=500$ (dashed), and $R_{S}=1000 \Omega$ (dotted). The parameters are similar to the ones used in Fig. 2 . The full arrows point to the gap value $\Delta$ estimated from the transition temperature $T_{c}$ using the standard BCS relation $\Delta$ $=1.76 k_{B} T_{c}$, whereas the open arrows point to $\Delta=1.9 k_{B} T_{c}$, where the factor 1.9 is known for bulk $\mathrm{Nb}$.

cies were measured for several other samples.

We also measured the differential resistance $d V / d I$, which is shown as a function of voltage $V$ in Fig. 4. What actually was measured is $d V / d I$ as a function of bias current $I$. This data was converted to the displayed voltage dependence by integration. Similar to the temperature dependence, $d V / d I$ has a nonmonotonic dependence. It first drops for increasing voltage and shows a minimum (a dip) before increasing again at higher voltages. The dip occurs close to the gap value $\Delta$ of the superconductor. $\Delta$ is estimated from the apparent transition temperature $T_{c}=7.5 \mathrm{~K}$ of the junction using the zero-temperature BCS relation $\Delta=1.76 k_{B} T_{c}$, yielding $\Delta$ $=1.14 \mathrm{meV}$ (black arrows). The agreement is even better if we use instead of the BCS factor of 1.76 for the ratio $\Delta / k_{B} T_{c}$ the factor 1.9, which is the reported ratio for bulk $\mathrm{Nb}$. This yields $\Delta=1.23 \mathrm{meV}$ (open arrows). Similar to $R(T)$, we used the BTK model to calculate the differential resistance, which is shown as a solid curve. The dashed and dotted curves correspond as before to the BTK model including a classical resistor in series. The theoretical curves display very pronounced dips at $\pm \Delta$, which are apparently strongly damped in the measurements. Unlike in the temperature dependent case, i.e., $R(T)$, the series-resistor model improves the agreement only marginally. In particular the strong dips are not removed.

\section{B. Shot-noise measurements}

We measured the shot noise from the superconductor to one of the normal reservoirs of the submicrometer beam splitter as schematically shown in Fig. 1(c). The measurement yields $S_{V_{1} V_{2}}=S_{V}$ as a function of bias current $I$. To obtain the intrinsic current noise $S_{I}(I)$ of the junction, Eq. (1) is applied using the calibration parameters as we have described it in the experimental part of Sec. II. The result is shown in Fig. 5. It corresponds to the same sample, for which $R(T)$ and $d V / d I(V)$ have been shown in Figs. 3 and 4, respectively. 


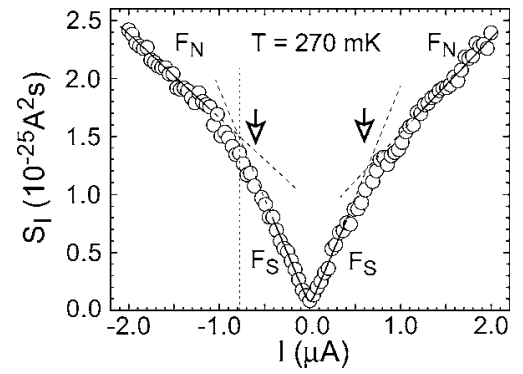

FIG. 5. Power spectral density $S_{I}$ of the current noise of a submicrometer S-N junction as a function of applied current $I . S_{I}$ is extracted from the measured voltage noise $S_{V_{1}, V_{2}}$ between the superconductor and one of the normal reservoirs [see Fig. 1(c)] according to the Eq. (1). A clear crossover from a large Fano factor $F_{S}$ at small bias currents to a reduced Fano factor $F_{N}$ for large currents is observed. This crossover coincides with gap $\Delta$ of the superconductor (open arrows).

The full temperature and voltage dependence of the power-spectral density $S_{I}$ of the current fluctuations can only be expressed in a simple analytical form for a junction with a constant channel transmission coefficient $\mathcal{T}$. It is given by ${ }^{35}$

$$
S_{I}=\frac{4(1-F) k_{B} T}{R}+F \cdot 2 e I \operatorname{coth}\left(\frac{e V}{2 k_{B} T}\right),
$$

where $F$ is known as the Fano factor and equals $1-\mathcal{T}$.

Noise measurements are generally analyzed in two limiting cases: (a) for small applied voltages $e V \ll k_{B} T$, for which $S_{I}$ equals the Johnson-Nyquist equilibrium noise (the thermal noise) $4 k_{B} T / R$, and (b) for large applied voltages $e V \gg k_{B} T$, for which a linear dependence of $S_{I}(I)$ is expected. In the limit of shot noise, i.e., the latter case, $S_{I}=F \cdot 2 e I$ and it is the Fano factor $F$, which is the central parameter that is deduced from such measurements. ${ }^{1,5,7} F=1$ for a junction in which all channels have low transmission eigenvalues, i.e., in tunnel junctions. ${ }^{5}$ In electronic devices in which charge is transported by single electrons alone, the Fano factor can in general be written as $F=\Sigma_{n} \mathcal{T}_{n}\left(1-\mathcal{T}_{n}\right) / \Sigma \mathcal{T}_{n}$, which is always smaller or at most equal to one. Hence, the suppression of shot noise in mesoscopic devices has been a central focus of research during recent years. For a review we refer to Refs. 1 and 21 . In contrast to "normal" conducting devices, enhancement of shot noise has recently been found in superconducting devices, in (S-I-S) (Ref. 22) and S-N junctions, ${ }^{30,31}$ as well as in superconducting S-N-S links. ${ }^{27,28}$ The two extreme cases of S-N junctions are the tunnel junction and the ballistic junction. In the former, the noise in the superconducting state is doubled $\left(F_{S}=2\right)$ as compared to the normal state $\left(F_{N}=1\right){ }^{22,31}$ In the latter, shot noise disappears completely, i.e., $F_{S}=F_{N}=0$.

The doubling of the shot noise in the superconducting state may be interpreted as being caused by the effective charge $e^{\star}$ of the charge carriers, ${ }^{8,24,31-33}$ which are Cooper pairs with $e^{\star}=2 e$, provided the temperature and the applied voltage are sufficiently small. One has to emphasize that the doubling of the shot noise is not generic. ${ }^{46}$ For a single channel S-N junction with transparency $\mathcal{T}$, the ratio of the Fano factors in the superconducting and normal state equals $F_{S} / F_{N}=8 /(2-\mathcal{T})^{2}$, which is always larger than 2 . It only equals 2 in the tunneling regime. If there are many channels with a distribution of eigenvalues $\mathcal{T}_{n}$, there is a doubling from $F_{N}=1 / 3$ to $F_{S}=2 / 3$ in the diffusive case, ${ }^{30,34,67}$ but $F_{N}=1 / 4$ (Refs. 18 and 20) increases to $F_{S}=0.604$ in case of an open chaotic cavity with a superconducting and normal terminal. The ratio in this case is $F_{S} / F_{N}=2.4$. Whereas the doubling of shot noise in $\mathrm{S}-\mathrm{N}$ devices has been stressed and confirmed in the tunneling and diffusive regime, a ratio of $F_{S} / F_{N}>2$, which should be the rule rather than the exception in few-channel conductors, has not been reported before.

The measured shot noise in Fig. 5 clearly displays two regimes in which $S_{I}(I)$ is nearly linear. In the low-current (low-voltage) regime, the slope is larger than in the highcurrent (high-voltage) regime. The crossover on the positive $(I>0)$ and negative $(I<0)$ side of the curve occurs at $\approx 0.62$ and $\approx-0.78 \mu A$, corresponding to a voltage of $\approx 1.3$ and $\approx-1.5 \mathrm{mV}$, in reasonable agreement with the value of the superconducting gap parameter $\Delta / e=1.23 \mathrm{mV}$ (open arrows), which we have deduced before. The agreement is good on the positive side, but somewhat off on the negative side, where the crossover appears to be shifted to a larger value. Asymmetries in the crossover as well as in the Fano factors were seen in other samples too. The low- and highbias slopes are identified with $F_{S}$ (low currents) and $F_{N}$ (high currents). We deduce $F_{S}=0.58 \pm 0.10$ and $F_{N}=0.25 \pm 0.04$ (average of slopes for $I<0$ and $I>0$ ). We note that the values of the Fano factors are considerably suppressed as compared to the case of a weakly transparent $\mathrm{S}-\mathrm{N}$ junction. We also see that the experimental results yields a ratio of the Fano factors $F_{S} / F_{N}=2.3$, which is indeed larger than two.

In contrast to conductivity measurements, from which the average transmission probability can be deduced, measurements of the shot noise provide insight into the actual distribution of the transmission eigenvalues, which helps to find the correct description of the scattering problem of the actual device. By making use of all measured parameters, the resistance in the normal and superconducting state, as well as $F_{S}$ and $F_{N}$, different models will be compared in detail in the last section.

Finally, shot noise measurements were also performed in a perpendicular magnetic field $B$, see Fig. 6 . It is seen that the separation in two regimes, characterized by distinct Fano factors, disappears around $B=3 \mathrm{~T}$, corresponding to the critical field of the $\mathrm{Nb}$ contact, which was measured independently. Figure 6 also shows that not only the Fano factor $F_{S}$ in the superconducting state is suppressed, a decrease, though a smaller one, is also observed in the normal state for $F_{N}$. The origin is likely due to magnetic-field induced suppression of backscattering in the semiconductor nanostructure, a well-known phenomenon in mesoscopic physics. ${ }^{68}$ This observation proves that scattering is taking place within the beam splitter in zero magnetic field adding up with the finite transparency of the superconductor-2DEG interface to the whole scattering problem. If we assume that ideally transmitting edge states have formed at the highest field, the superconductor-2DEG interface would have to account for the remaining Fano factor of $F_{N}=0.16$ alone, yielding a transparency of as much as $\mathcal{T}=1-F_{N}=0.84$ in a single chan- 


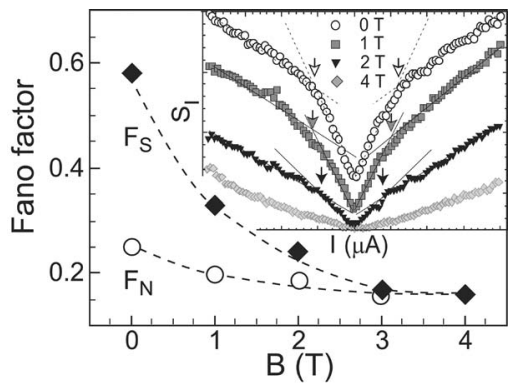

FIG. 6. Fano factors versus perpendicular magnetic field $B$ (symbols). The dashed curves are guides to the eyes. As $B$ is increased, the enhancement of the Fano factor in the superconducting relative to the normal state (i.e., the ratio $F_{S} / F_{N}$ ) diminishes and fully disappears for $B \geq 3 \mathrm{~T}$. Note, that $F_{N}$ also slightly decreases as the field increases. Inset: The power spectral density $S_{I}$ of the current noise as a function of the bias current $I$ for $B=0,1,2$, and $4 \mathrm{~T}$. The curves are shifted vertically for clarity. The crossover (arrows) between the superconducting and normal state shifts to lower voltages for increasing magnetic field as expected.

nel model. From reference transport measurements on Hall bars we know that the longitudinal resistance of the 2DEG displays pronounced magnetic-field-induced oscillations (Shubnikov-de Haas oscillations) for $B \leq 3 \mathrm{~T}$. Though the resistance minima do not yet reach zero, clear quantum Hall plateaus are discernible, see Fig. 1(b). At 4 T, for example, the Hall measurements show that ten Landau levels are occupied. Hence, the number of edge channels is already smaller than the number of transporting channels in zero magnetic field, which was estimated from the width of the constrictions to be 19. Since, transport follows the edges in the quantum Hall regime, the $84 \%$ transmission at the S-N interface must be seen as an upper bound for the respective transmission probability in zero magnetic field.

In order to deepen our understanding of both the resistance and the shot noise data, we need to thoroughly compare our data with a model consisting of a S-N contact with finite transparency to which an additional scattering region is added.

\section{DISCUSSION AND MODELING}

We compare the data of one device with a set of models. These models are schematically shown in Fig. 7. Figure 7(a) is the wire model with a fixed number of channels $N$. Ideal contacts are assumed for the superconductor on the left and normal metal on the right side. The barrier, which may form in the processing of the $\mathrm{Nb}$ contact to the InAs 2DEG is captured by a tunneling barrier with transparency $\Gamma$. A disordered region, parametrized by its length $L$ and elastic scattering mean-free path $l_{e}$, can be included. Figure 7(b) is the cavity model. Here, both sides can have different numbers of channels. This is in fact closer to the real device geometry where the contact on the $\mathrm{Nb}$ side is wider than the constrictions at the $\mathrm{Y}$ branch. The contact on the right is always assumed to be "open," meaning that its conductance is equal to $N G_{0}$, where $G_{0}=2 e^{2} / h$ is the quantum conductance, whereas there may be a tunneling barrier in the left contact in (a)

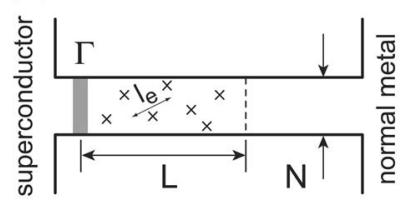

(b)

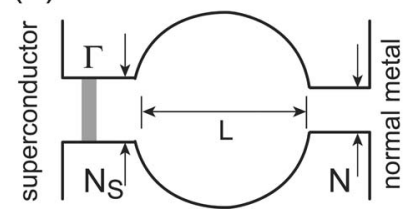

FIG. 7. Illustration of the two basic models which we have used to analyze our data. (a) is based on a wire and (b) on a cavity. The models are considered in different regimes, but always in the limit of zero temperature. In (a) we distinguish between the ballistic $\left(l_{e}\right.$ $\gg L)$, intermediate $\left(l_{e} \sim L\right)$, and diffusive $\left(l_{e} \ll L\right)$ regime, whereas in (b) the cavity is assumed to be either open on both sides (no barrier) or only open on one side with a tunneling barrier on the other side, described by its transparency $\Gamma$.

order to model the effective transparency of the N-S contact. In the following when we refer to "the normal state" we consider the N-N case in which the superconductor is in the normal state. Similarly, when we refer to "the superconducting state" we consider the S-N case. Note that in contrast to the real device all the models have two terminals only. This simplification is likely to introduce deviations, because the open third terminal will add dephasing. However, neglecting dephasing (relaxation in general) facilitates the comparison with theory greatly. Now, we can use the machinery of mesoscopic physics to calculate the conductances and shotnoise Fano factors in the normal and superconducting state. It is one of the great hallmarks of mesoscopic physics that these quantities can be calculated in the coherent transport regime if the distribution $\rho(\mathcal{T})$ of transmission eigenvalues $\mathcal{T}$ for the particular device is known.

At zero temperature $T=0$ the respective equations for the conductances $G_{(N, S)}$ and shot-noise powers $S_{(N, S)}$ in the normal $(N)$ and superconducting $(S)$ state $\operatorname{are}^{69}$

$$
\begin{gathered}
G_{N}=G_{0} N \int_{0}^{1} d \mathcal{T} \rho(\mathcal{T}) \mathcal{T} \\
G_{S}=G_{0} N \int_{0}^{1} d \mathcal{T} \rho(\mathcal{T}) \frac{2 \mathcal{T}^{2}}{(2-\mathcal{T})^{2}} \\
S_{N}=S_{0} N \int_{0}^{1} d \mathcal{T} \rho(\mathcal{T}) \mathcal{T}(1-\mathcal{T}), \\
S_{S}=S_{0} N \int_{0}^{1} d \mathcal{T} \rho(\mathcal{T}) \frac{16 \mathcal{T}^{2}(1-\mathcal{T})}{(2-\mathcal{T})^{4}},
\end{gathered}
$$

where $S_{0}=2 e V G_{0}$ and $N$ is the total number of conducting channels in the system. Even more so, general concepts have been developed allowing to calculate the distribution function $\rho(\mathcal{T})$ for all models shown in Fig. $7 .^{70}$

The result of this comparison is summarized in Table I. In the following we will go sequentially through the models and discuss the assumptions and results. We focus on the quantities $G_{(N, S)}$ and $F_{(N, S)}$ at zero temperature. In case of the simplest models we will also compare with the full temperature 
TABLE I. Comparison of the measured data, i.e., the linear conductance $G_{(N, S)}$ and the shot-noise Fano factors $F_{(N, S)}$ in the normal $(N)$ and superconducting $(S)$ state with various models. Schematics for the models are shown in Figs. 7(a) and 7(b).

\begin{tabular}{|c|c|c|c|c|c|c|c|}
\hline & Measured & $\begin{array}{c}L \ll l_{e} \\
\text { Ballistic (BTK) }\end{array}$ & Ballistic with $R_{S}{ }^{\mathrm{a}}$ & $\begin{array}{c}L \gg l_{e} \\
\text { Diffusive }\end{array}$ & Open chaotic cavity & $\begin{array}{c}L \sim l_{e} \\
\text { Quasi-ballistic }\end{array}$ & Chaotic cavity with barrier \\
\hline Fig. 7 & & a & a & a & $\mathrm{b}$ & a & $\mathrm{b}$ \\
\hline$G_{N} / G_{0}$ & $6.1 \pm 0.05$ & 6.1 & 6.1 & 5.8 & 5.4 & 6.1 & 6.1 \\
\hline$G_{S} / G_{0}$ & $5.5 \pm 0.05$ & 5.5 & 5.5 & 5.8 & 6.4 & 5.5 & 5.6 \\
\hline$F_{N}$ & $0.25 \pm 0.04$ & 0.27 & 0.16 & 0.33 & 0.25 & 0.36 & 0.33 \\
\hline$F_{S}$ & $0.58 \pm 0.10$ & 1.34 & 0.80 & 0.67 & 0.60 & 0.77 & 0.84 \\
\hline$\Gamma$ & & 0.73 & 0.72 & 1 & 1 & 0.55 & 0.7 \\
\hline$N$ & & 9 & 11 & $19^{\mathrm{b}}$ & 11 & 17 & 11 \\
\hline
\end{tabular}

${ }^{\text {a Series resistance, } R_{S}=500 \Omega \text {. }}$

${ }^{b}$ The number of channels is fixed by the geometry, i.e., $N \sim 2 w / \lambda_{F}$.

dependence of the conductance $G(T)$ and the voltage dependence of the differential conductance $d I / d V(V)$. The parameters $G_{S}$ and $G_{N}$ are deduced in the experiment from the linear-response conductance measured at the smallest temperature $270 \mathrm{mK}$ and at $\sim 8 \mathrm{~K}$, respectively.

The simplest possible model to compare with is a $\mathrm{S}-\mathrm{N}$ junction in which the normal part is ballistic. This problem was considered by Blonder, Tinkham, and Klapwijk and is known as the BTK model. ${ }^{65}$ In the BTK model of a S-N interface, the junction is characterized by a single transmission coefficient, i.e., $\rho(\mathcal{T})=\delta(\mathcal{T}-\Gamma)$. For $\Gamma=1$, the junction resistance decreases with decreasing temperature and the conductance is doubled at $T=0 \mathrm{~K}$ due to Andreev reflection. In the opposite limit $\Gamma \ll 1$, Andreev reflection is suppressed and the resistance increases monotonically with decreasing temperature below $T_{c}$. The comparison of the equations for $G_{N}$ and $G_{S}$ with the experimental values yields $\Gamma=0.73$ and $N \approx 9$. We can now use these two parameters to calculate the full temperature and the nonlinear voltage dependence of the conductance and compare both with the measurements. This is shown in Figs. 3 and 4 where the calculated curves are the solid ones. Figure 3 shows the temperature dependence of the linear two-terminal resistance $R(T)$ and Fig. 4 the differential resistance $d V / d I$ as a function of voltage $V$, measured at $T=270 \mathrm{mK}$. As imposed by this procedure the measured (circles) and calculated (solid) curves in Fig. 3 match at zero temperature and at (or above) $T_{c}$ in the normal state. Similarly, the measured (circles) and calculated (dashed) curves in Fig. 4 match at zero bias and approximately at the largest bias voltage of $|V|=4 \mathrm{mV}$ at which one closely approaches the normal state. In the intermediate temperature and voltage regime substantial deviations are found. The theory predicts a much larger conductance increase in the intermediate regime than is seen in the experiment. This is particularly striking in the differential resistance where a strong dip (or a peak in the conductance) is expected to occur near the superconducting gap $\Delta$.

The experimentally observed strong damping of this conductance peak near the superconducting gap has also been seen in other work. ${ }^{62,71}$ It can be caused by pair breaking due to inelastic scattering. Even more so, the shape of the quasiparticle density-of-state in the vicinity of $\pm \Delta$, which acquires singularities in the BCS model, may strongly be damped at the interface between the $\mathrm{Nb}$ and the $2 \mathrm{DEG} .{ }^{62}$ The reason for the latter may be a disordered interface caused by sputter cleaning or by partial oxidation. For the former, we suspect that the second terminal of the Y branch, which has been left open, is a source of dephasing. Electrons at the $Y$ branch can scatter into the drain contact, but may also be scattered into the third terminal, from which they are reflected back but with unknown phase. In addition, the large deviations in the intermediate regime may also stem from the assumed model, which is likely to be too simple. We will come back to this issue when we refine the model. Let us now see whether the ballistic BTK model can capture the shot-noise results, i.e., the measured Fano factors. In case of an N-N barrier, the Fano factor is given by $F_{N}=1-\Gamma$. The estimated $\Gamma=0.73$ predicts $F_{N}=0.27$, which is consistent with the measured shot-noise Fano factor of 0.25 . In the superconducting state, however, the theory for a $\mathrm{S}-\mathrm{N}$ barrier ${ }^{69}$ predicts $F_{S}=8(1$ $-\Gamma) /(2-\Gamma)^{2}=1.34$, whereas the measured Fano factor is substantially smaller and amounts to 0.58 only. We may also do the reverse and deduce the transparency $\Gamma$ from the measured Fano factors instead. $F_{S}=0.58$ then implies $\Gamma=0.91$ which is both inconsistent with the measured Fano factor in the normal state $F_{N}$ and with the temperature dependence of the resistance in Fig. 3. Hence, the ballistic junction model does not yield consistent values. This is not surprising, because of the structured beam splitter in front of the superconductor. Each arm of the splitter is comprised of a relatively narrow opening. Hence, parts of the eigenchannels emanating from the Nb-2DEG interface must be back reflected at these exit ports. This results in an additional voltage drop, i.e., in an additional resistance. Since the sample is likely to be coherent, this resistance cannot simply be treated as a classical series resistor. The whole structure composed of the $\mathrm{S}-\mathrm{N}$ interface, cavity, and exit leads need to be treated as one scattering problem. We will discuss this latter on, but still try the classical series resistor model as an additional test case next.

Fits to the measured two-terminal resistance $R(T)$ and $d V / d I(V)$ including a classical resistor $R_{S}$ in series to the S-N interface are shown in Figs. 3 and 4 for two values of $R_{S}$, i.e., $R_{S}=500$ and $R_{S}=1000 \Omega$. It turns out that if $R_{S}$ is increased, 
the fit of $R(T)$ improves in the intermediate temperature regime. However, the width of the zero-bias peak in $d V / d I$ broadens with increasing $R_{S}$, so that the agreement gets worse here. A reasonable compromise is found for $R_{S}$ $=500 \Omega$. Using the conductance measurements we deduce a junction transparency of $\Gamma=0.72$ and obtain for the number of channels $N \approx 11$ within this model. Because the series resistor is a classical one it does not contribute to nonequilibrium shot noise. In order to deduce the Fano factor the current fluctuations $S_{I}$ have to be plotted versus current $I$. $S_{I}$ is obtained from the measured voltage fluctuations by dividing $S_{V}$ with the total resistance $R=R_{S N}+R_{S}$ squared. In the framework of this model this division is incorrect. Instead, one should divide by $R_{S N}^{2}$, only. This now yields a correction factor amounting to $\left(1+R_{S} / R_{S N}\right)^{2}$, which has to be applied to the measured data. For ease of comparison, we apply the inverse $1 /\left(1+R_{S} / R_{S N}\right)^{2} \approx 0.60$ to the model calculation. As a result, the predicted Fano factor in the superconducting state $F_{S}=0.80$ is getting closer to the measured value, but $F_{N}$ $=0.16$ is now clearly too small as compared with the measured value. Adding a classical series resistance improves somewhat the agreement between the experiment and model of $R(T)$. It also relaxes slightly the large discrepancy of the Fano factor in the superconducting state. However, it is clear that this model is an oversimplification, because the device is more than just one junction with a single transparency and the whole device, including the cavity and beam splitter should be treated on equal footing.

A fixed transparency is a very idealized assumption, one which never holds true in a practical multichannel device. There are many reasons why a distribution of transparencies has to be considered: The junction interface is never perfectly homogeneous, the sample has been structured and the boundaries may be rough on the scale of the Fermi wavelength and there are dopants within the heterostructure. It is possible that the quality of the 2DEG was degraded near the $\mathrm{S}-\mathrm{N}$ interface during the sample processing, for example, due to the Ar sputtering of the MESA prior to $\mathrm{Nb}$ deposition. ${ }^{62} \mathrm{In}$ addition, the narrow constrictions defining the output ports must be seen as a scattering center. If we assume that disorder is substantial, we are led to the diffusive regime, which is another limiting case contrasting with the ballistic junction limit discussed before. For a diffusive conductor, the distribution of transmission eigenvalues $\rho(\mathcal{T})$ is given by a universal result $1 /(2 s \mathcal{T} \sqrt{1-\mathcal{T}})$, where $s=L / l_{e} \cdot{ }^{12}$ Using this distribution function yields $G_{S} / G_{N}=1,{ }^{72} F_{N}=1 / 3,{ }^{12}$ and $F_{S}$ $=2 / 3 .^{32}$ As can be seen from the table, the agreement is much better, in particular for the Fano factors, suggesting that elastic scattering must be considered. However, the measured conductances are not equal in the normal and superconducting state, i.e., $G_{S} / G_{N}=1$, as predicated by this model. Though the agreement is much better, this model is an oversimplification too. We know that the scattering-mean-free path in the bulk of the 2DEG is much larger than the size of the nanostructure which is considered here. In addition, the magnetic-field dependence of the Fano factor $F_{N}$ in the normal state (Fig. 6) is inconsistent with a diffusive conductor. One should therefore rather view the device as a cavity with three terminals: a wide $\mathrm{Nb}$ one, and two narrow leads defined by the constriction. This justifies comparing our data also to an open chaotic cavity.

We only compare our data in Table I with the symmetric cavity, because this is suggested by the measured Fano factor in the normal state, which is found to be close to $F_{N}=0.25$. A suppression factor of $1 / 4$ is the expected result for the symmetric open cavity. ${ }^{18,20,21}$ The distribution of transmission eigenvalues $\rho(\mathcal{T})$ for a chaotic cavity, contacted by two open leads each having $N$ ideally transmitting channels, is given by another bimodal distribution function $1 / \pi \sqrt{\mathcal{T}(1-\mathcal{T})} \cdot{ }^{18,69}$ Using Eqs. (3)-(6) yields: $G_{N} / G_{0}=N / 2, G_{S} / G_{0}=(2-\sqrt{2}) N$, $F_{N}=0.25$, and $F_{S}=0.6036$. As can be seen from Table I, the measured Fano factors compare very well with this model. On the other hand, this model predicts $G_{S}>G_{N}$, whereas $G_{S}<G_{N}$ in the experiment. We mention that $G_{S}>G_{N}$ also holds if the cavity is allowed to be asymmetric. In fact, $G_{S} / G_{N}$ is minimal for the symmetric cavity and reaches the well-known factor of two for strong asymmetries. This shows that we cannot cure the deficiency in the conductances between theory and model just by tuning the asymmetry alone. In an attempt to lower $G_{S}$ as compared to $G_{N}$ we now further try to refine our model. There are two refinements we can consider: We may start from the "universial" diffusive case and ask the question what happens if the elastic scattering mean-free path $l_{e}$ is increased up to the point when $l_{e}$ becomes of the order of the device size. Second, we may add additional scattering by adding a barrier to one side of the open cavity.

We first consider the "quasi-ballistic" case studied by de Jong and Beenakker. ${ }^{32,69}$ In their model of a S-N device, a tunnel barrier is inserted (which may be used to model the quality of the contact itself) in series to a disordered region of length $L$ in which the elastic scattering length is $l_{e}$. de Jong and Beenakker were able to study the crossover from the ballistic to the diffusive regime for an arbitrary ratio of $s=L / l_{e}$. We have already considered the limiting cases $s=0$, which is the ballistic BTK limit, and the universal diffusive case $s \rightarrow \infty$. Interesting for us is the intermediate case $s \sim 1$, which can be computed for both the normal and the superconducting state using the scaling theory of the generalized conductance. $^{32,67}$ The numerical calculation yields $\Gamma=0.55$ and $N=17$ for $s \sim 1$. de Jong and Beenakker also showed that the shot noise power can vary between zero and twice the Poisson value, depending on the junction parameters. ${ }^{32}$ Using $\Gamma=0.55$ and $N=17$, we obtain for the Fano factors $F_{N}$ $=0.36$ and $F_{S}=0.77$.

In view of the real device geometry, a refinement of the open cavity model is appealing too. The real device is asymmetric in that the width of the contact at the $\mathrm{Nb}$ side is wider than the constrictions at the exits. In addition, there is likely a barrier at the interface of the 2DEG and the superconductor, the transparency of which has been denoted by $\Gamma$ in the previous models. The simplest way to calculate $\rho(\mathcal{T})$ is to apply circuit theory ${ }^{70}$ to the series connection of a tunnel junction with a quantum-point contact (QPC). The tunnel junction is the element at the $\mathrm{Nb}$ side. It is parameterized by its conductance $G_{t}$. The QPC models the narrow constriction on the right side. It is parameterized by its conductance $G$ $=\left(2 e^{2} / h\right) N$, i.e. by the number of (open) channels. Though $\Gamma$ 
does not appear in the model explicitly (only the ratio $G / G_{t}$ enters), it can be extracted from the fitted value which we obtain for $G_{t}$. $G_{t}$ can be expressed as $G_{0} \Gamma N w_{S} / w_{N}$, where $w_{S, N}$ is the width of the 2DEG at the $\mathrm{S}$ and at the $\mathrm{N}$ side, respectively. In trying to find the best match, we fix the conductance in the normal state to the measured value and vary $N$ to get the best agreement with all measured parameters. This approach yields $N=11, \Gamma=0.7, F_{N}=0.33$, and $F_{S}=0.84$.

Let us summarize the results of all the models. One may say that none yields perfect agreement in all four measured parameters, i.e., $G_{N}, G_{S}, F_{N}$, and $F_{S}$. The most realistic ones in terms of the actual geometry, i.e., the quasi-ballistic and cavity with barrier models, yield reasonable agreement in all parameters. The Fano factors are predicted to be slightly larger than measured. In fact, this trend holds true for all models considered. The measured Fano factors are systematically smaller. We suspect that the origin for this discrepancy is found in the third terminal, i.e., the second outgoing lead of the Y branch, which was left open in the measurements of the conductance and noise. Electrons entering into this lead will relax and thermalize before being re-injected into the device again. Relaxation in general reduces shot noise. ${ }^{35,73-76}$ With regard to the number of channels the different models predict $N=9, \ldots, 17$ for the channel number in the constriction. This is in fair agreement with an estimate of the channel number based on the lithographic width and the Fermi wavelength, yielding $N \sim 19$. It is quite reasonable that the channel number deduced electrically turns out to be somewhat smaller, because of depletion in the vicinity of the MESA after etching.

\section{CONCLUSIONS}

In summary, we have realized a mesoscopic superconductor-normal beam splitter geometry in a solid state hybrid system and characterized its electrical properties using two-terminal measurements. We can account for both the conductance and shot noise data by modeling the device as a highly transparent S-N interface connected in series with a "short" scattering region, which is in the quasi-ballistic transport regime. The scattering region is formed by the cavity in the 2DEG between the S-N interface and the two constrictions forming the electron beam-splitter. The shot noise measured across the superconductor and one arm of the beam splitter is enhanced relative to the normal state. The respective Fano factors are in reasonable agreement with the Landauer description (scattering problem) of coherent transport. In particular the ratio of the Fano factors $F_{S} / F_{N}$ is experimentally found to be larger than two, showing that the doubling of shot noise is not a generic property of S-N devices. Residual deviations, in particular in the vicinity of the gap energy in the differential conductance measurements, are likely due to relaxation, a source of which is the second arm of the beam splitter which was left open in the reported experiments. Current fluctuations can be suppressed by an extra terminal, even in the absence of a net (average) current.

Our devices are very well suited to explore positive cross-correlations, ${ }^{39}$ as have recently been predicted in several theoretical papers. ${ }^{39-42,49,50}$ Of these theoretical treatments, Ref. 42 is in closest correspondence with our experiments. In Ref. 42, an electron cavity is connected to one superconducting and two normal leads via point contacts. Positive correlations are predicted to appear for a dominant coupling to the superconducting lead. The devices which we have studied in this work have roughly similar couplings to the $\mathrm{S}$ and $\mathrm{N}$ leads. In the next step, one has to make use of the ability of semiconductors to tune the transparency of the constrictions with additional electrodes (split gates), which can be fabricated self-aligned with the etched trenches. This would greatly help in the search for positive correlations in solid-state nanostructures.

\section{ACKNOWLEDGMENTS}

We thank G. Burkard. M. Gräber, P. Recher, P. Samuelsson, and C. Strunk for their fruitful discussions and acknowledge contributions to this work by T. Nussbaumer. This work has been supported by the Swiss NFS, the NCCR on Nanoscience, and by the BBW (RTN "DIENOW" under the fifth framework EU program).

\footnotetext{
*Electronic address: Christian.Schoenenberger@unibas.ch

${ }^{1}$ For a general and comprehensive introduction, see: C. W. J. Beenakker and C. Schönenberger, Phys. Today 56, 37 (2003).

${ }^{2}$ L. Saminadayar, D. C. Glattli, Y. Jin, and B. Etienne, Phys. Rev. Lett. 79, 2526 (1997).

${ }^{3}$ R. de-Picciotto, M. Reznikov, M. Heiblum, V. Umansky, G. Bunin, and D. Mahalu, Nature (London) 389, 162 (1997).

${ }^{4}$ M. Reznikov, R. de Picciotto, T. G. Griffiths, M. Heiblum, and V. Umansky, Nature (London) 399, 238 (1999).

${ }^{5}$ H. Birk, M. J. M. de Jong, and C. Schönenberger, Phys. Rev. Lett. 75, 1610 (1995).

${ }^{6}$ A. Kumar, L. Saminadayar, D. C. Glattli, Y. Jin, and B. Etienne, Phys. Rev. Lett. 76. 2778 (1996).

${ }^{7}$ M. Reznikov, M. Heiblum, H. Shtrikman, and D. Mahalu, Phys. Rev. Lett. 75, 3340 (1995).
}

${ }^{8}$ V. A. Khlus, Sov. Phys. JETP 66, 1243 (1987).

${ }^{9}$ G. B. Lesovik, JETP Lett. 49, 592 (1989).

${ }^{10}$ M. Buttiker, Phys. Rev. Lett. 65, 2901 (1990).

${ }^{11}$ M. Henny, S. Oberholzer, C. Strunk, and C. Schönenberger, Phys. Rev. B 59, 2871 (1999).

${ }^{12}$ C. W. J. Beenakker and M. Büttiker, Phys. Rev. B 46, R1889 (1992).

${ }^{13}$ K. E. Nagaev, Phys. Lett. A 169, 103 (1992).

${ }^{14}$ F. Liefrink, J. I. Dijkhuis, M. J. M. de Jong, L. W. Molenkamp, and H. van Houten, Phys. Rev. B 49, 14066 (1994).

${ }^{15}$ A. H. Steinbach, J. M. Martinis, and M. H. Devoret, Phys. Rev. Lett. 76, 3806 (1996).

${ }^{16}$ R. J. Schoelkopf, P. J. Burke, A. A. Kozhevnikov, D. E. Prober, and M. J. Rooks, Phys. Rev. Lett. 78, 3370 (1997).

${ }^{17}$ S. Oberholzer, E. Sukhorukov, and C. Schönenberger, Nature 
(London) 415, 765 (2002).

${ }^{18}$ R. A. Jalabert, J.-L. Pichard, and C. W. J. Beenakker, Europhys. Lett. 27, 255 (1994).

${ }^{19}$ Ya. M. Blanter and E. V. Sukhorukov, Phys. Rev. Lett. 84, 1280 (2000).

${ }^{20}$ S. Oberholzer, E. V. Sukhorukov, C. Strunk, C. Schönenberger, T. Heinzel, M. Holland, Phys. Rev. Lett. 86, 2114 (2001)

${ }^{21}$ Y. M. Blanter and M. Büttiker, Phys. Rep. 336, 1 (2000).

${ }^{22}$ P. Dieleman, H. G. Bukkems, T. M. Klapwijk, M. Schicke, and K. H. Gundlach, Phys. Rev. Lett. 793486 (1997).

${ }^{23}$ K. E. Nagaev and M. Büttiker, Phys. Rev. B 63, 081301(R) (2001).

${ }^{24}$ D. Averin and H. T. Imam, Phys. Rev. Lett. 76, 3814 (1996)

${ }^{25}$ R. Cron, M. F. Goffman, D. Esteve, and C. Urbina, Phys. Rev. Lett. 86, 4104 (2001).

${ }^{26}$ P. Roche, H. Perrin, D. C. Glattli, H. Takayanagi, and T. Akazaki, Physica C 352, 73 (2001).

${ }^{27}$ T. Hoss, C. Strunk, T. Nussbaumer, R. Huber, U. Staufer, C. Schönenberger, Phys. Rev. B 62, 4079 (2000).

${ }^{28}$ C. Hoffmann, F. Lefloch, and M. Sanquer, Eur. Phys. J. B 29, 629 (2002).

${ }^{29}$ B. Reulet, A. A. Kozhevnikov, D. E. Prober, W. Belzig, and Yu. V. Nazarov, Phys. Rev. Lett. 90, 066601 (2003).

${ }^{30}$ X. Jehl, M. Sanquer, R. Calemczuk, and D. Mailly, Nature (London) 405, 50 (2000).

${ }^{31}$ F. Lefloch, C. Hoffmann, M. Sanquer, and D. Quirion, Phys. Rev. Lett. 90, 067002 (2003).

${ }^{32}$ M. J. M. de Jong and C. W. J. Beenakker, Phys. Rev. B 49, 16070 (1994).

${ }^{33}$ B. A. Muzykantskii and D. E. Khmelnitskii, Phys. Rev. B 50, 3982 (1994).

${ }^{34}$ A. A. Kozhevnikov, R. J. Schoelkopf, L. E. Calvet, M. J. Rooks, and D. E. Prober, J. Low Temp. Phys. 118, 671 (2000).

${ }^{35}$ M. Büttiker, Phys. Rev. B 46, 12485 (1992).

${ }^{36}$ M. Henny, S. Oberholzer, C. Strunk, T. Heinzel, K. Ensslin, M. Holland, and C. Schönenberger, Science 284, 296 (1999).

${ }^{37}$ W. D. Oliver, J. Kim, R. C. Liu, and Y. Yamamoto, Science 284, 299 (1999)

${ }^{38}$ R. C. Liu, B. Odom, Y. Yamamoto, and S. Tarucha, Nature (London) 391, 263 (1998).

${ }^{39}$ M. P. Anantram and S. Datta, Phys. Rev. B 53, 16390 (1996).

${ }^{40}$ J. Torrès and T. Martin, Eur. Phys. J. B 12, 319 (1999).

${ }^{41}$ J. Börlin, W. Belzig, and C. Bruder, Phys. Rev. Lett. 88, 197001 (2002).

${ }^{42}$ P. Samuelsson and M. Büttiker, Phys. Rev. Lett. 89, 046601 (2002).

${ }^{43}$ M. Büttiker and P. Samuelsson, Physica E (Amsterdam) 18, 60 (2003).

${ }^{44}$ D. Loss and D. P. DiVincenzo, Phys. Rev. A 57, 120 (1998).

${ }^{45}$ D. P. DiVincenzo and D. Loss, Superlattices Microstruct. 23, 419 (1998).

${ }^{46}$ J. Torrès, T. Martin, and G. B. Lesovik, Phys. Rev. B 63, 134517 (2001)

${ }^{47}$ M.-S. Choi, C. Bruder, and D. Loss, Phys. Rev. B 62, R13569 (2000).

${ }^{48}$ P. Samuelsson and M. Büttiker, Phys. Rev. B 66, 201306(R) (2002).

${ }^{49}$ G. B. Lesovik, T. Martin, and G. Blatter, Eur. Phys. J. B 24, 287 (2001).
${ }^{50}$ P. Recher, E. V. Sukhorukov, and D. Loss, Phys. Rev. B 63, 165314 (2001).

${ }^{51}$ C. Bena, S. Vishveshwara, L. Balents, and M. P. A. Fisher, Phys. Rev. Lett. 89, 037901 (2002).

${ }^{52}$ P. Recher and D. Loss, Phys. Rev. B 65, 165327 (2002).

${ }^{53}$ V. Bouchiat, N. Chtchelkatchev, D. Feinberg, G. B. Lesovik, T. Martin, J. Torrès, Nanotechnology 14, 77 (2003).

${ }^{54}$ N. M. Chtchelkatchev, G. Blatter, G. B. Lesovik, and T. Martin, Phys. Rev. B 66, 161320(R) (2002).

${ }^{55}$ G. Burkard, D. Loss, E. V. Sukhorukov, Phys. Rev. B 61, R16303 (2000).

${ }^{56}$ D. Loss and E. V. Sukhorukov, Phys. Rev. Lett. 84, 1035 (2000).

${ }^{57}$ J. Nitta, T. Akazaki, H. Takayanagi, and K. Arai, Phys. Rev. B 46, 14286 (1992).

${ }^{58}$ H. Takayanagi, T. Akazaki, and J. Nitta, Phys. Rev. Lett. 75, 3533 (1995).

${ }^{59}$ T. Akazaki, H. Takayanagi, J. Nitta, and T. Enoki, Appl. Phys. Lett. 68, 418 (1996).

${ }^{60}$ Y. Takagaki, Phys. Rev. B 57, 4009 (1998).

${ }^{61}$ E. Toyoda, H. Takayanagi, and H. Nakano, Phys. Rev. B 59, R11653 (1999).

${ }^{62}$ K. Neurohr, A. A. Golubov, Th. Klocke, J. Kaufmann, T. Schäpers, J. Appenzeller, D. Uhlisch, A. V. Ustinov, M. Hollfelder, H. Lüth, and A. I. Braginski, Phys. Rev. B 54, 17018 (1996).

${ }^{63}$ D. C. Glattli, P. Jacques, A. Kumar, and L. Saminadayar, J. Appl. Phys. 81, 7350 (1997).

${ }^{64}$ The linear dependence of $S_{V}(T)-S_{\text {off }}$ with temperature $T$, which we used in the calibartion, is not exact, because $R$ is not constant, but temperature dependent as well. $R$ varies in this temperature range by $\pm \approx 10 \%$. For the calibration an average value for $R$ was used.

${ }^{65}$ G. E. Blonder, M. Tinkham, and T. M. Klapwijk, Phys. Rev. B 25, 4515 (1982).

${ }^{66}$ Y. Imry, Introduction to Mesoscopic Physics (Oxford University, New York, 1997).

${ }^{67}$ C. W. J. Beenakker, B. Rejaei, and J. A. Melsen, Phys. Rev. Lett. 72, 2470 (1994).

${ }^{68}$ C. W. J. Beenakker and H. van Houten, in Quantum Transport in Semiconductor Nanostructures in Solid State Physics, Vol. 44, edited by H. Ehrenreich and D. Turnbull (Academic, San Diego, 1991).

${ }^{69}$ M. J. M. de Jong and C. W. J. Beenakker, in Mesoscopic Electron Transport, edited by L. L. Sohn, L. P. Kouwenhoven, and G. Schön, NATO Advanced Studies Institute, Series E: Applied Sciences, (Kluwer, Dordrecht, 1997), Vol. 345.

${ }^{70}$ W. Belzig, A. Brataas, Y. V. Nazarov, and G. E. W. Bauer, Phys. Rev. B 62, 9726 (2000).

${ }^{71}$ M. Jakob, H. Stahl, J. Knoch, J. Appenzeller, B. Lengeler, H. Hardtdegen, and H. Lüth, Appl. Phys. Lett. 76, 1152 (2000).

${ }^{72}$ C. W. J. Beenakker, Phys. Rev. B 46, R12841 (1992).

${ }^{73}$ M. L. Roukes, M. R. Freeman, R. S. Germain, R. C. Richardson, and M. B. Ketchen, Phys. Rev. Lett. 55, 422 (1985).

${ }^{74}$ F. C. Wellstood, C. Urbina, and J. Clarke, Phys. Rev. B 49, 5942 (1994).

${ }^{75}$ K. E. Nagaev, Phys. Rev. B 52, 4740 (1995).

${ }^{76}$ M. Henny, H. Birk, R. Huber, C. Strunk, A. Bachtold, M. Krüger, and C, Schönenberger, Appl. Phys. Lett. 71, 773 (1997). 